\title{
Revascularisation led to less angina and fewer adverse cardiac events than did optimal medical care in angina pectoris in the elderly
}

The TIME Investigators. Trial of invasive versus medical therapy in elderly patients with chronic symptomatic coronary-artery disease (TIME): a randomised trial. Lancet 2001 Sep 22;358:951-7.

QUESTION: In older patients with at least Canadian Cardiac Society (CCS) class II angina pectoris, is an invasive strategy of left heart catheterisation followed by either percutaneous coronary intervention $(\mathrm{PCl})$ or coronary artery bypass grafting (CABG) more effective than a strategy of optimised medical treatment?

\section{Design}

Randomised (allocation concealed*), unblinded,* controlled trial with 6 months of follow up.

\section{Setting}

$\{14$ university and non-university hospitals $\} \uparrow$ in Switzerland.

\section{Patients}

305 patients who were $\geqslant 75$ years of age (mean age $80 y$, $56 \%$ men) with at least CCS class II angina pectoris despite treatment with $\geqslant 2$ antianginal drugs. Exclusion criteria included acute myocardial infarction (MI) within the previous 10 days, concomitant valvular or other heart disease, predominant congestive heart failure, and lifelimiting comorbid disease. 4 patients (2 from each group) were not included in the analysis.

\section{Intervention}

155 patients were allocated to an invasive strategy consisting of coronary angiography followed by PCI or CABG if feasible. 150 patients were allocated to optimising medical treatment consisting of an increase in the number or dose of antianginal drugs.

\section{Main outcome measures}

The primary end points were quality of life and major adverse cardiac events (death, non-fatal MI, or admission to hospital for an acute coronary syndrome).

\section{Main results}

Analysis was by intention to treat. At 6 months, quality of life increased in both treatment groups but data for the paired analysis were available for $<80 \%$ of those randomised. Angina severity and number of anginal medications taken decreased to a greater extent, and major adverse cardiac events occurred less frequently in those allocated to the invasive strategy (table).

\section{Conclusion}

Revascularisation led to less angina and fewer major adverse cardiac events than did optimal medical care in patients $\geqslant 75$ years of age with at least class II angina pectoris despite treatment with $\geqslant 2$ antianginal drugs.

*See glossary.

$\dagger$ Information provided by the author.

Invasive treatment $v$ optimised medical care in chronic angina

\begin{tabular}{llll} 
Outcomes at 6 months & Invasive & Optimised & Mean difference $(95 \%$ Cl) \\
\hline Mean change from baseline in angina class & -2.0 & -1.6 & $-0.4(-0.72$ to -0.08$)$ \\
\hline Mean change in number of antianginal medications & -1.0 & -0.2 & $-0.8(-1.09$ to -0.51$)$ \\
\hline & & & RRR (Cl) \\
Major adverse cardiac events & $19 \%$ & $49 \%$ & $61 \%(44$ to 73$)$ \\
\hline
\end{tabular}

¥Abbreviations defined in glossary; RRR, NNT, and Cl calculated from data in article.

\section{COMMENTARY}

The advantage of direct or catheter based revascularisation over medical treatment in the general population with advanced forms of coronary disease is well documented. ${ }^{1}$ The TIME trial sought to determine whether these results could be reliably applied to elderly patients when the risk associated with intervention is greater.

The design of the study allowed considerable latitude to determine which strategy would be used in the invasive group. This latitude probably accurately reflects common treatment scenarios in many centres; however, several important points should be noted. A bias against surgery is suggested by the small number of patients assigned surgery (33 of 147 patients) despite 88 having 3-vessel disease and 21 having left-main disease. Furthermore, the "intensification" of care in the 150 patients assigned to optimal medical treatment resulted in the addition of $<1(0.8)$ antianginal medication per patient, and only about half $(55 \%)$ of the patients had additional increases in drug dosages.

Notwithstanding possible bias against surgery and the limited opportunity for medication optimisation, the invasive group fared much better at 6 months. Event free survival in these patients was higher in the invasive group $(81 \%)$ than the medical group $(51 \%)(\mathrm{p}<0.001)$. This information is useful for those treating the elderly, and it parallels the 22\% relative risk reduction in a younger group of patients reported in the Fragmin and Fast Revascularisation during Instability in Coronary Artery Disease (FRISC II) trial. ${ }^{2}$ These data support early angiography and invasive treatment in stable elderly patients with class II or greater angina. 